\title{
How to prevent the microvascular complications of type 2 diabetes beyond glucose control
}

In this Clinical Review (BMJ 2017;356:i6505, doi:10.1136/bmj. i6505), the term "hypoglycemia," which originally appeared several times in the figure should have read "hyperglycemia." This has now been corrected. 